\title{
Characterization of the MCRred2 form of methyl-coenzyme $M$ reductase: a pulse EPR and ENDOR study
}

\section{Journal Article}

Author(s):

Finazzo, Cinzia; Harmer, Jeffrey; Jaun, Bernhard; Duin, Evert C.; Mahlert, Felix; Thauer, Rudolf K.; Doorslaer, Sabine Van; Schweiger, Arthur

Publication date:

2003-05

Permanent link:

https://doi.org/10.3929/ethz-b-000055668

Rights / license:

In Copyright - Non-Commercial Use Permitted

Originally published in:

JBIC Journal of Biological Inorganic Chemistry 8(5), https://doi.org/10.1007/s00775-003-0450-y 


\section{Cinzia Finazzo · Jeffrey Harmer · Bernhard Jaun Evert C. Duin · Felix Mahlert · Rudolf K. Thauer Sabine Van Doorslaer · Arthur Schweiger \\ Characterization of the MCR $_{\text {red2 }}$ form of methyl-coenzyme $M$ reductase: a pulse EPR and ENDOR study}

Received: 3 October 2002 / Accepted: 1 February 2003/Published online: 6 March 2003

(c) SBIC 2003

\begin{abstract}
Methyl-coenzyme M reductase (MCR), which catalyses the reduction of methyl-coenzyme $\mathrm{M}\left(\mathrm{CH}_{3}-\mathrm{S}\right.$ $\mathrm{CoM}$ ) with coenzyme $\mathrm{B}$ (H-S-CoB) to $\mathrm{CH}_{4}$ and $\mathrm{CoM}-\mathrm{S}-$ $\mathrm{S}-\mathrm{CoB}$, contains the nickel porphinoid $\mathrm{F} 430$ as prosthetic group. The active enzyme exhibits the Ni(I)derived axial EPR signal $\mathrm{MCR}_{\text {red1 }}$ both in the absence and presence of the substrates. When the enzyme is competitively inhibited by coenzyme M (HS-CoM) the $\mathrm{MCR}_{\text {red1 }}$ signal is partially converted into the rhombic EPR signal $\mathrm{MCR}_{\mathrm{red} 2}$. To obtain deeper insight into the geometric and electronic structure of the red2 form, pulse EPR and ENDOR spectroscopy at X-and Q-band microwave frequencies was used. Hyperfine interactions of the four pyrrole nitrogens were determined from ENDOR and HYSCORE data, which revealed two sets of nitrogens with hyperfine couplings differing by about
\end{abstract}

Electronic Supplementary Material Supplementary material is available for this article if you access the article at http://dx.doi. org/10.1007/s00775-003-0450-y. A link in the frame on the left on that page takes you directly to the supplementary material.

C. Finazzo $\cdot$ J. Harmer $\cdot$ S. Van Doorslaer $\cdot$ A. Schweiger $(\square)$ Physical Chemistry, ETH Zurich,

8093 Zurich, Switzerland

E-mail: schweiger@phys.chem.ethz.ch

Fax: + 41-1-6321538

B. Jaun

Organic Chemistry, ETH Zurich,

8093 Zurich, Switzerland

E.C. Duin · F. Mahlert · R.K. Thauer

Max-Planck-Institut für terrestrische Mikrobiologie

and Laboratorium für Mikrobiologie, Fachbereich Biologie,

Philipps-Universität, Karl-von-Frisch-Strasse,

35043 Marburg, Germany

Present address: S. Van Doorslaer

Spectroscopy in Biophysics and Catalysis (SIBAC Laboratory), University of Antwerp, Universiteitsplein 1,

2610 Wilrijk, Belgium

Present address: E.C. Duin

Department of Chemistry,

Auburn University, Auburn,

AL, 36849-5312, USA a factor of two. In addition, ENDOR data enabled observation of two nearly isotropic ${ }^{1} \mathrm{H}$ hyperfine interactions. Both the nitrogen and proton data indicate that the substrate analogue coenzyme $\mathrm{M}$ is axially coordinated to $\mathrm{Ni}(\mathrm{I})$ in the $\mathrm{MCR}_{\text {red2 } 2}$ state.

Electronic Supplementary Material Supplementary material is available for this article if you access the article at http://dx.doi. org/10.1007/s00775-003-0450-y. A link in the frame on the left on that page takes you directly to the supplementary material.

Keywords ENDOR - HYSCORE - Factor 430 . Methanogenic archaea $\cdot$ Nickel enzyme

Abbreviations $\mathrm{CH}_{3}-\mathrm{S}$-CoM: methyl-coenzyme M • $d q$ : double-quantum $\cdot E N D O R$ : electron nuclear double resonance $E P R$ : electron paramagnetic resonance $H S$-CoB: coenzyme B $\cdot H S$-CoM: coenzyme $\mathrm{M}$. HYSCORE: hyperfine sublevel correlation $\cdot M C R$ : methyl-coenzyme $\mathrm{M}$ reductase $\cdot M C R_{\text {red } 1}$ : MCR exhibiting the red1 EPR signal $\cdot M C R_{\text {red2 }}: \mathrm{MCR}$ exhibiting the red2 EPR signal $\cdot s q$ : single-quantum

\section{Introduction}

Methyl-coenzyme $\mathrm{M}$ reductase (MCR) catalyzes the reduction of methyl-coenzyme $\mathrm{M}\left[\mathrm{CH}_{3}-\mathrm{S}-\mathrm{CoM}\right.$, 2-(methylthio)ethanesulfonate] with coenzyme B (HS$\mathrm{CoB}$, 7-thioheptanoylthreonine phosphate) to methane and CoM-S-S-CoB, which is the methane-forming step in the energy metabolism of all methanogenic archaea [1]. The enzyme contains the nickel porphinoid cofactor F430, which has been identified as the prosthetic group of MCR [2, 3, 4]. The crystal structure of inactive MCR reveals the presence of two symmetry-related active sites, each containing one cofactor F430 in an inactive and EPR-silent $\mathrm{Ni}(\mathrm{II})$ state $\left(\mathrm{d}^{8}, S=1\right)[5,6,7$, 8]. Different forms of the enzyme and interconversions between various MCR species are known [9, 10]. Several of them, including the states $\mathrm{MCR}_{\mathrm{ox} 1}, \mathrm{MCR}_{\mathrm{ox} 2}$, 
$\mathrm{MCR}_{\mathrm{red} 1}$, and $\mathrm{MCR}_{\mathrm{red} 2}$, and various subgroups of these forms, are EPR visible. The oxidation state of all the red forms is $\mathrm{Ni}(\mathrm{I})[11,12,13]$. The active form, $\mathrm{MCR}_{\text {red1 }}$, exhibits an axial Ni-based EPR spectrum characteristic of a $\mathrm{d}^{9}, S=1 / 2 \mathrm{Ni}(\mathrm{I})$ complex with the unpaired electron in the $\mathrm{d}_{x 2-y 2}$ orbital and hyperfine structure due to the four pyrrole nitrogen ligands. $\mathrm{MCR}_{\text {red1 }}$ is converted into $\mathrm{MCR}_{\mathrm{red} 2}$ in the presence of coenzyme M (HS-CoM, 2-mercaptoethanesulfonate) and coenzyme $\mathrm{B}$, the $\mathrm{MCR}_{\text {red2 }}$ signal apparently being generated at the expense of the $\mathrm{MCR}_{\mathrm{red} 1}$ signal. At most, $50 \%$ of $\mathrm{MCR}_{\text {red1 }}$ is found to be converted to $M R_{\text {red2 }}$. EPR data show clearly that $M_{C R}$ red2 is nickel based. First, the CW EPR spectrum of ${ }^{61} \mathrm{Ni}-$ labeled $\mathrm{MCR}_{\text {red2 }}$ is considerably broadened due to a ${ }^{61} \mathrm{Ni}$ hyperfine interaction of $\left(A_{1}, A_{2}, A_{3}\right)=(39,44$, 67) $\mathrm{MHz}[9,10]$. Second, the g matrix is highly rhombic, indicating that the unpaired electron is in a low-symmetry environment. Third, the large deviation of the principal $g$ values from the $g$ value of the free electron is typical for a metal-based complex with a large spin-orbit coupling. In contrast to $\mathrm{MCR}_{\text {red1 }}$ and $\mathrm{MCR}_{\mathrm{oxl}}$, the ligand hyperfine interactions are not resolved in the X-band or Q-band EPR spectra of $\mathrm{MCR}_{\text {red2. }}$. The poor resolution of the EPR spectra can be traced back to $g$ and/or $A$ strain caused by the environment surrounding the nickel ion not being as well defined as in the other EPR-active MCR species. It is thus necessary to use high-resolution techniques such as HYSCORE (hyperfine sublevel correlation) and pulse ENDOR (electron nuclear double resonance) to obtain further information about the coordination sphere.

In this contribution we report on the magnetic parameters of the ligand nuclei of $\mathrm{MCR}_{\text {red2, in }}$ particular the hyperfine and nuclear quadrupole interactions of the "corphin" nitrogens and on proton hyperfine interactions, which have been determined to obtain a deeper insight into the geometric and electronic structure of this state of MCR. A combination of HYSCORE and pulse ENDOR [14] experiments carried out at $\mathrm{X}$ - and Q-band frequencies enabled determination of the hyperfine interactions of the four pyrrole nitrogens, which differ in their hyperfine couplings by a factor of two. In addition, two large and nearly isotropic ${ }^{1} \mathrm{H}$ hyperfine couplings were observed. We tentatively assign these couplings to protons of the substrate analogue coenzyme $\mathrm{M}$, which we assume to be axially bound.

\section{Materials and methods}

\section{Sample preparation}

The sample preparation is described elsewhere [9]. Note that a sample containing only $\mathrm{MCR}_{\text {red2 }}$ cannot be prepared, since a significant amount of $\mathrm{MCR}_{\mathrm{red} 1}$ is always present. It is possible, however, to prepare a sample that only contains $\mathrm{MCR}_{\text {red1 }}$.
Spectroscopy

The X-band pulse ENDOR experiments were carried out on a Bruker ELEXSYS spectrometer [microwave (mw) frequency $9.73 \mathrm{GHz}$ ] equipped with a helium gas-flow cryostat (Oxford). All measurements were done at $15 \mathrm{~K}$ with a repetition rate of $200 \mathrm{~Hz}$. Pulse measurements at Q-band (mw frequency $35.3 \mathrm{GHz}$ ) were performed on a home-built spectrometer [15] at $20 \mathrm{~K}$ with a repetition rate of $1 \mathrm{kHz}$.

Davies-ENDOR [16] spectra at X- and Q-band frequencies were measured with the mw pulse sequence $\pi-T-\pi / 2-\tau-\pi-\tau$-echo. At $\mathrm{X}$-band, short mw pulses of length $t_{\pi / 2}=26 \mathrm{~ns}$ and $t_{\pi}=52 \mathrm{~ns}$ were used to suppress signals from weakly coupled protons that overlap with the strongly coupled nitrogen signals. A time interval $\tau=900 \mathrm{~ns}$ was used, and during time $T$ an $\mathrm{rf} \pi$ pulse of length $5.4 \mu \mathrm{s}$ with variable frequency $\mathrm{v}_{\mathrm{ENDOR}}$ and frequency increments of $50 \mathrm{kHz}$ was applied. At Q-band, proton (nitrogen) spectra were measured with mw pulse lengths of $t_{\pi / 2}=50$ (30) ns and $t_{\pi}=100$ (60) ns, and a time interval $\tau=220 \mathrm{~ns}$. An $\mathrm{rf} \pi$ pulse with a length of 18 (29) $\mu$ s and frequency increments of $50 \mathrm{kHz}$ was applied during time $T$.

HYSCORE [17], with the pulse sequence $\pi / 2-\tau-\pi / 2-t_{1}-\pi-t_{2}-\pi /$ 2 - $\tau$-echo, is a two-dimensional experiment, which correlates nuclear frequencies in one electron spin manifold to nuclear frequencies in the other electron spin manifold. Measurements at Q-band were run using the following parameters: mw pulses of length $t_{\pi / 2}=t_{\pi}=16 \mathrm{~ns}$, starting times of $96 \mathrm{~ns}$ for $t_{1}$ and $t_{2}$, and time increments $\Delta t=8 \mathrm{~ns}$ (data matrix $256 \times 256$ ). To increase the modulation depth, the second and the third $\pi / 2$ pulses were replaced by a matched pulse of length 24 ns [18]. To avoid blind spots, spectra with $\tau$ values of $112,132,152$, and $192 \mathrm{~ns}$ were recorded. An eight-step phase cycle was used to remove unwanted echoes.

Both ENDOR and HYSCORE experiments were carried out at different magnetic field positions to collect data from different molecular orientations (orientation selectivity) [14].

\section{Data manipulation}

The data were processed with the program MATLAB 6.1 (MathWorks, Natick, Mass., USA). The time traces of the HYSCORE spectra were baseline corrected with a third-order polynomial, apodized with a Hamming window and zero filled. After a two-dimensional Fourier transformation the absolute-value spectra were calculated. The HYSCORE spectra recorded with different $\tau$ values were added to eliminate $\tau$-dependent blind spots. The Davies-ENDOR spectra were simulated with the program EasySpin (http:// www.esr.ethz.ch) and the HYSCORE spectra with a program written in-house [19].

\section{Theory}

The spin Hamiltonian for a spin system with a $\mathrm{Ni}(\mathrm{I})$ ion (electronic configuration $\left.3 \mathrm{~d}^{9}, S=1 / 2\right),{ }^{14} \mathrm{~N}(I=1)$, and ${ }^{1} \mathrm{H}(I=1 / 2)$ nuclei in frequency units is given by [14]:

$H=\left(\beta_{\mathrm{e}} / h\right) \mathbf{S g} \mathbf{B}_{0}+\sum \mathbf{S} \mathbf{A}_{i} \mathbf{I}_{i}-\left(\beta_{\mathrm{n}} / h\right) \sum g_{i, \mathrm{n}} \mathbf{I}_{i} \mathbf{B}_{\mathbf{0}}+\sum \mathbf{I}_{i} \mathbf{Q}_{i} \mathbf{I}_{i}$

where the terms describe the electron Zeeman interaction, the hyperfine interactions, the nuclear Zeeman interactions, and the nuclear quadrupole interactions (for nuclei with $I>1 / 2$ ). The principal values $Q_{1}, Q_{2}$, and $Q_{3}$ of the traceless $\mathbf{Q}_{\mathrm{i}}$ tensor are usually expressed in terms of the quadrupole coupling constant $K=\mathrm{e}^{2} \mathrm{qQ}$ $4 I(2 I-1) h$ and the asymmetry parameter $\eta=\left(Q_{1}-Q_{2}\right) / Q_{3}$, with $Q_{1}=-K(1-\eta), Q_{2}=-K(1+\eta)$, and $Q_{3}=2 K$.

The ENDOR spectrum of a nucleus with spin $I=1 / 2$ consists of two transitions. For $\mathbf{B}_{0}$ along one of the hyperfine principal axes the frequencies are given by: 
$v=\left|v_{I} \pm \frac{A_{K}}{2}\right|$

where $v_{I}$ is the nuclear Zeeman frequency and $A_{K}$ is one of the principal hyperfine values. For $v_{I}>\left|A_{K} / 2\right|$ (weak coupling case) the two lines are centered at $v_{I}$ and separated by $A_{K}$. In $\mathrm{MCR}_{\mathrm{red} 2}$ this is the case for the proton signals observed at X- and Q-band.

For a nucleus with spin $I=1\left({ }^{14} \mathrm{~N}\right)$ the ENDOR spectrum consists of four lines. For the magnetic field along one of the principal axes the frequencies are given by:

$v=\left|v_{I} \pm \frac{A_{K}}{2} \pm \frac{3}{2} Q_{K}\right|$

For frequencies along arbitrary field orientations, see [14]. For $v_{\mathrm{I}}<\left|A_{K} / 2\right|$ (strong coupling case) the lines are centered at $A_{K} / 2$ and split by $2 v_{I}$ and the nuclear quadrupole interaction.

In a HYSCORE experiment on a nucleus with spin $I=1$ in each of the two electron spin manifolds $(\alpha, \beta)$ there exist three transitions between the nuclear sublevels, two single-quantum (sq) transitions with $\left|\Delta m_{I}\right|=1$, and one double-quantum (dq) transition with $\left|\Delta m_{I}\right|=2$. The two-dimensional HYSCORE spectra are usually dominated by cross-peaks between the dq frequencies.

\section{Results}

The Q-band EPR spectra of $\mathrm{MCR}_{\mathrm{red} 1}$ and $\mathrm{MCR}_{\mathrm{red} 2}$ (and simulations) are shown in Fig. 1. The spectrum of $\mathrm{MCR}_{\text {red1 }}$ overlaps with the spectrum of $\mathrm{MCR}_{\text {red2 }}$, except for the $g_{1}$ feature of $\mathrm{MCR}_{\mathrm{red} 2}$ at the low-field end. The spectrum of $\mathrm{MCR}_{\text {red2 }}$ exhibits strong rhombicity with $g$ values $\left(g_{1}, g_{2}, g_{3}\right)=(2.2869,2.2313,2.1753)$. These values are more accurate than the X-band data [9] because of the higher resolution afforded by Q-band data (see Table 1).

Figure 2A and Fig. 2B show ENDOR spectra recorded at X-band (at a low-field position where only $\mathrm{MCR}_{\mathrm{red} 2}$ exists) and at Q-band (field positions indicated in Fig. 1), respectively. At X-band, signals from weakly coupled protons, which overlap with signals from the strongly coupled nitrogen(s), are suppressed by using short mw pulses (hyperfine contrast selective ENDOR [14]). The spectrum is thus dominated by signals from strongly coupled nitrogens and signals from two protons with a large hyperfine coupling (indicated by asterisks). In Fig. 2B (Q-band) the spectra arise only from nitrogens since the protons are shifted to higher frequencies (around $48 \mathrm{MHz}$; see Fig. 4). The low-field ENDOR spectrum (trace Ia) contains only $\mathrm{MCR}_{\text {red2, }}$ whereas traces IIa and IIIa contain contributions from both

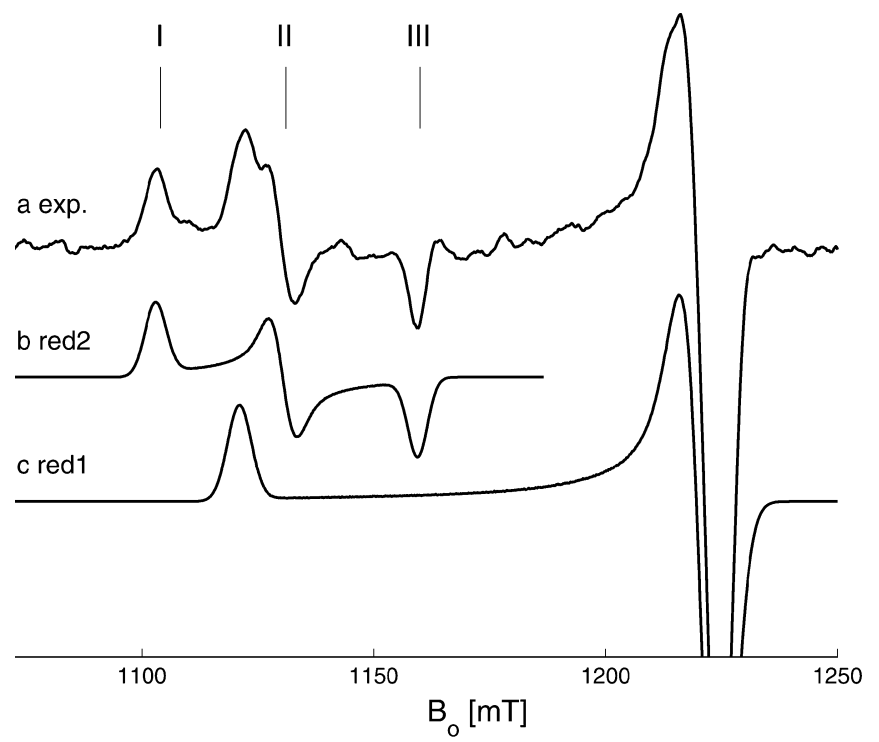

Fig. 1 (a) First derivative of the Q-band FID-detected EPR spectrum measured at $20 \mathrm{~K}$. (b) Simulation of $\mathrm{MCR}_{\mathrm{red} 2}$ and (c) $\mathrm{MCR}_{\mathrm{red} 1}$. The labels $I(1103.2 \mathrm{mT}), I I(1130.2 \mathrm{mT})$, and III $(1155.7 \mathrm{mT}, 1157.7 \mathrm{mT})$, indicate the field positions used in the ENDOR and HYSCORE experiments, respectively

$\mathrm{MCR}_{\text {red1 }}$ and $\mathrm{MCR}_{\text {red2 }}$. Traces IIc and IIIc were measured on a separate sample containing only the $\mathrm{MCR}_{\text {red } 1}$ species, which allows the features originating from $\mathrm{MCR}_{\text {red } 1}$ in traces IIa and IIIa to be identified. Simulations of both the X-and Q-band ENDOR spectra were achieved by considering three ${ }^{14} \mathrm{~N}$ nuclei: two equivalent nitrogens with $A_{\text {iso }}=24.6 \mathrm{MHz}$ and one nitrogen with $A_{\text {iso }}=22.5 \mathrm{MHz}$ (see Table 2). At the low-field position the simulations of the individual components are given: Id $(\times 1$ nitrogen), Ic $(\times 2$ equivalent nitrogens $)$, and $\mathrm{Ib}$ the sum. For trace Id a stick diagram is also given, showing how the nitrogen frequencies are determined by the hyperfine, nuclear Zeeman, and nuclear quadrupole interactions. In a powder sample, broad lines rather than sharp peaks are generally observed since many molecular orientations contribute to the spectrum at each field position; this makes interpretation without simulation difficult (in particular, trace IIa, measured along $\mathbf{g}_{2}$ ). However, spectra recorded at the edges of the EPR spectrum (traces Ia and IIIa for $\mathrm{MCR}_{\mathrm{red} 2}$ ) are "single-
Table $1 g$ values of $\mathrm{MCR}_{\mathrm{red} 2}$ in comparison with other MCR forms and $\mathrm{Ni}(\mathrm{I})$ complexes

\begin{tabular}{|c|c|c|c|c|}
\hline Complex & $g_{1}$ & $g_{2}$ & $g_{3}$ & Ref. \\
\hline $\mathrm{MCR}_{\mathrm{red} 2}(\mathrm{Q}$-band) & $2.2869(5)$ & $2.2313(5)$ & $2.1753(5)$ & This work \\
\hline $\mathrm{MCR}_{\mathrm{red} 2}(\mathrm{X}$-band) & 2.2880 & 2.2348 & 2.1790 & {$[9]$} \\
\hline $\mathrm{MCR}_{\mathrm{red} 1 \mathrm{c}}$ & 2.250 & 2.071 & 2.0605 & [9] \\
\hline $\mathrm{MCR}_{\mathrm{red} 1}$ & 2.2485 & 2.070 & 2.060 & [20] \\
\hline $\mathrm{Ni}(\mathrm{I}) \mathrm{F} 430$ & 2.244 & 2.063 & 2.063 & [26] \\
\hline $\mathrm{MCR}_{\mathrm{ox} 1}$ & 2.2310 & 2.1667 & 2.1532 & [9] \\
\hline $\mathrm{MCR}_{\mathrm{ox} 1}$ & 2.229 & 2.166 & 2.148 & [20] \\
\hline $\mathrm{Ni}(\mathrm{I})(\mathrm{STPP})\left(\mathrm{SO}_{2}\right)$ & 2.075 & 2.087 & 2.187 & [23] \\
\hline $\mathrm{Ni}(\mathrm{I})(\mathrm{STPP})\left(2,4-\mathrm{Me}_{2} \mathrm{py}\right)_{2}$ & 2.262 & 2.233 & 2.131 & {$[23]$} \\
\hline$\left[\mathrm{Ni}(\mathrm{I})(\mathrm{DAPA})(\mathrm{SPh})_{2}\right]^{-}$ & 2.283 & 2.201 & 2.164 & [24] \\
\hline (CTPP) $\mathrm{Ni}(\mathrm{III}) \mathrm{OH}$ & 2.274 & 2.190 & 2.109 & {$[25]$} \\
\hline
\end{tabular}



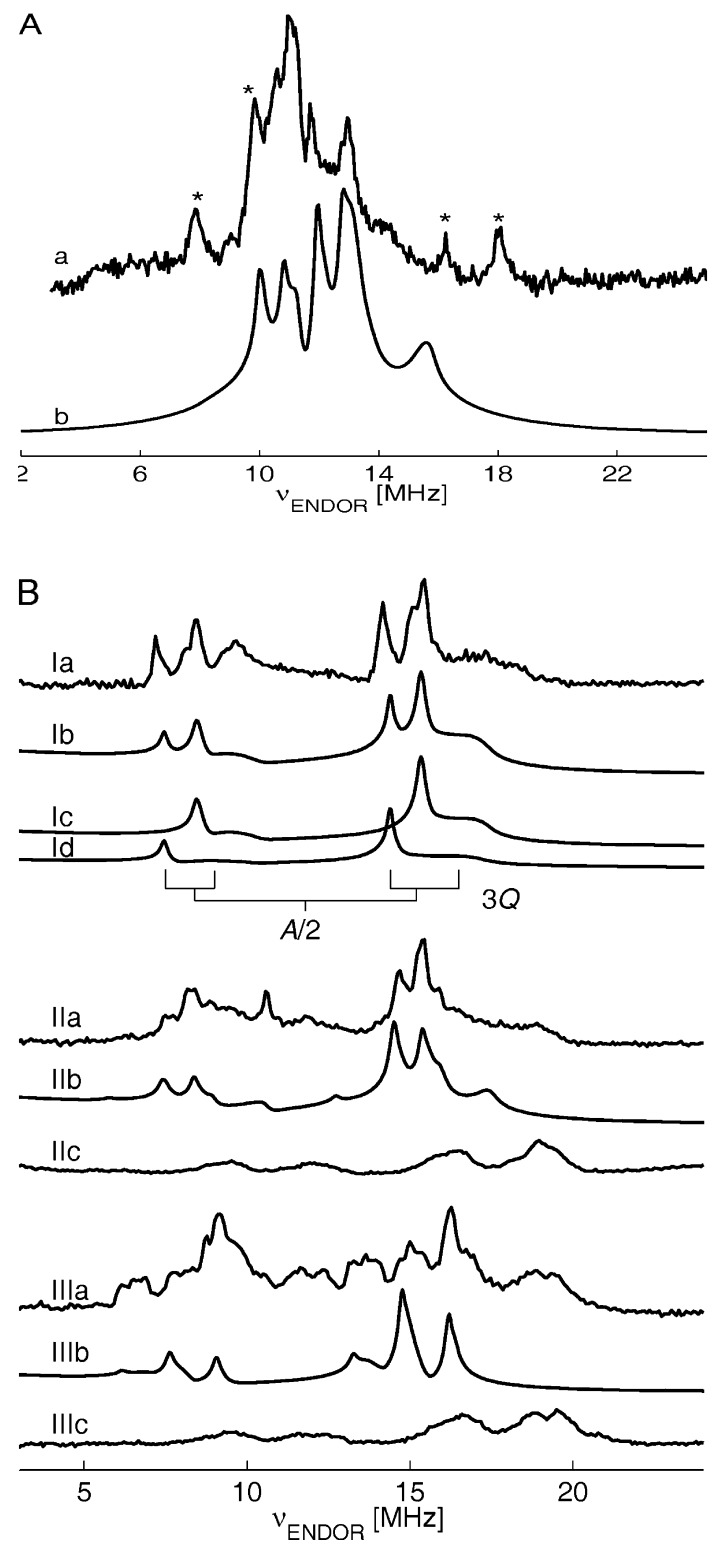

Fig. 2 A X-band Davies-ENDOR spectrum recorded at $303.7 \mathrm{mT}$ : (a) experimental, (b) nitrogen simulation. B Q-band nitrogen Davies-ENDOR spectra recorded at the observer positions indicated in Fig. 1. Ia, II $a$, and IIIa, experimental; $I b, I c, I d, I I b$, and $I I I b$, simulation; IIc and IIIc, experimental spectra of a sample containing only $\mathrm{MCR}_{\text {red } 1}$

crystal" like since they select only a narrow range of molecular orientations; the peaks here are thus sharper and amendable to a simple interpretation via a stick diagram. The complete set of ENDOR spectra and simulations are available as Supplementary material.

Q-band HYSCORE spectra measured at the observer positions indicated in Fig. 1 are shown in Fig. 3A. These spectra clearly reveal the presence of an additional nitrogen(s) with a smaller hyperfine coupling ( $A_{\text {iso }}$ $=13.8 \mathrm{MHz}$ ), which was barely observable in the ENDOR spectra. The nitrogens with the larger hyperfine couplings could also be observed in the HYSCORE spectra after matching the mw pulses to increase the modulation depth. In Fig. 3A, selected cross-peaks from the two types of ${ }^{14} \mathrm{~N}$ nuclei have been labeled. The peaks are predominantly found in the $(-,+)$ quadrant, indicating that for both types of nitrogens we have a strongcoupling situation, $A>2 v_{I} \approx 7 \mathrm{MHz}$. The dq cross-peaks in particularly are informative since along a principal axis they are centered at $A$, split by four times the ${ }^{14} \mathrm{~N}$ nuclear Larmor frequency $\left(v_{I} \approx 3.5 \mathrm{MHz}\right)$ and free from nuclear quadrupole broadening (to first order). In Fig. 3AII the dq cross-peaks from the weaker coupled nitrogens are centered around $(-20.6,7.0) \mathrm{MHz}$ and $(-7.0,20.6) \mathrm{MHz}$, and from the stronger coupled nitrogens around $(-31.2,17.4) \mathrm{MHz}$ and (-17.4, 31.2) $\mathrm{MHz}$. At the observer position in the middle of the EPR spectrum (Fig. 3AII), many molecular orientations contribute to the HYSCORE spectrum and thus ridges whose length reflects the anisotropy of the coupling parameters are observed. The HYSCORE spectra recorded at the edges of the EPR spectrum (Fig. 3AI and Fig. 3AIII for the $\mathrm{MCR}_{\text {red2 }}$ species) are "single-crystal" like and the peaks are thus sharper. At field positions outside the EPR spectrum of $\mathrm{MCR}_{\text {red2 }}$ the features from the nitrogen(s) with the smallest hyperfine coupling disappear, and only strongly coupled nitrogen signals remain consistent with the nitrogen couplings of $\mathrm{MCR}_{\mathrm{red} 1}$ given in [20]. Note that the diagonal peaks in the first quadrant are caused by an improper transfer of nuclear coherences between the two electron-spin manifolds by the $\pi$ pulse and slight phase errors in the phase cycling procedure. In Fig. 3B, a simulation for observer position II (along $\mathbf{g}_{2}$ ) is given which includes three stronger $\left(A_{\text {iso }}=24.6,24.6,22.5 \mathrm{MHz}\right)$ and one weaker coupled $\left(A_{\text {iso }}=13.8 \mathrm{MHz}\right)$ nitrogen (see Table 2 ). Simulations at the three field positions are given in the Supplementary material.

Figure 4 shows proton Davies-ENDOR spectra measured at Q-band; the observer positions are again those given in Fig. 1. Protons with nearly isotropic hyperfine couplings of $\left(A_{1}, A_{2}, A_{3}\right)=(10.1,10.7$, 10.3) $\mathrm{MHz}$ and $\left(A_{1}, A_{2}, A_{3}\right)=(6.5,7.1,6.6) \mathrm{MHz}$ are found at the three observer positions. These proton signals are also observed in the X-band ENDOR spectrum given in Fig. 2A, and they disappear when the observer position is moved outside the EPR spectrum of $\mathrm{MCR}_{\text {red2 } 2}$. In addition, a number of weakly coupled protons from the macrocycle and possibly from axial ligands with hyperfine couplings $\leq 4 \mathrm{MHz}$ are observed. An assignment of these smaller couplings to particular protons is not possible with the available (powder) data.

\section{Discussion}

The pulse EPR data show that $\mathrm{MCR}_{\mathrm{red} 2}$ is distinguished by two sets of nitrogen ligands with hyperfine couplings that differ by about a factor of two. In the ENDOR experiments, nitrogens with hyperfine coupling in the range $20.2-26.6 \mathrm{MHz}$ are observed, whereas in the HYSCORE spectra a nitrogen(s) with a 

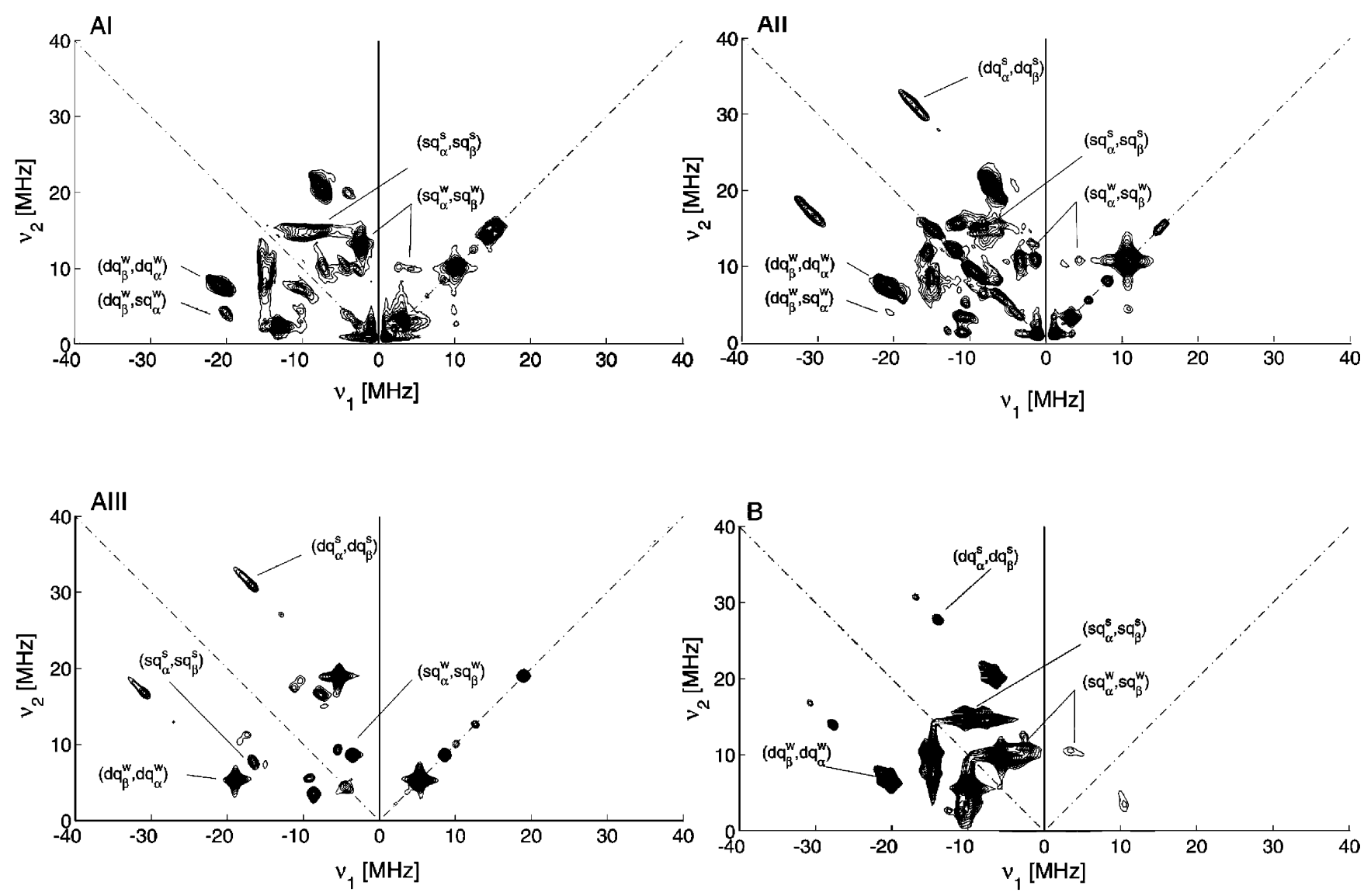

Fig. 3 A Q-band nitrogen HYSCORE spectra measured at the observer positions $I, I I$, and $I I I$ (see Fig. 1); B simulation for field position II. The cross-peaks are labeled in the following way: $s q$ (single-quantum) and $d q$ (double-quantum) frequencies in the $\alpha$ or $\beta$ electron spin manifolds; superscripts refer to the nitrogens with strong $(s)$ and weak $(w)$ hyperfine couplings

hyperfine coupling in the range $11.8-16.0 \mathrm{MHz}$ is identified. The two vastly different nitrogen couplings indicate that the macrocycle is significantly distorted, a finding which is corroborated by the large rhombicity of the $\mathbf{g}$ matrix.

Many rhombic five- and six-coordinated nickel(I) and nickel(III) complexes are described in the literature [21, 22], but in most of them the smallest $g$ value is very close to $g_{\mathrm{e}}=2.0023$, and the average $g$ value, $g_{\mathrm{av}}=\left(g_{1}+g_{2}+\right.$ $\left.g_{3}\right) / 3$, is much smaller than the one found for $\mathrm{MCR}_{\text {red2 } 2}$.

$g$ values similar to those found in $\mathrm{MCR}_{\mathrm{red} 2}$ have been reported, for example, for some $\mathrm{Ni}(\mathrm{I})(\mathrm{STPP})$ complexes (STPP $=5,10,15,20$-tetraphenyl-21-thiaporphyrin), where the nickel is coordinated to three nitrogens and one sulfur ligand of the macrocycle [23], for the pentacoordinated nickel complex [Ni(I)(DAPA)(SPh $\left.)_{2}\right]$; DAPA $=2,6$-bis[1-(phenylimino)ethyl]pyridine, with two thiolate ligands in the equatorial plane and three nitrogens of the DAPA ligand occupying the remaining coordination sites [24], and for $\mathrm{Ni}(\mathrm{III})$ tetraphenylcarboporphyrin, $\mathrm{Ni}(\mathrm{III})(\mathrm{CTPP})$, an inverted porphyrin where the nickel ion is coordinated to three nitrogens and one carbon in the porphyrin plane [25] (see Table 1).
In the case of $\mathrm{Ni}(\mathrm{I})(\mathrm{STPP})$ it has been found that the rhombicity of the EPR spectra is mainly caused by the axial ligands, rather than by a replacement of one of the pyrroles by a thiophene. The $\mathrm{g}$ matrix of the complex $\mathrm{Ni}(\mathrm{I})(\mathrm{STPP})\left(\mathrm{SO}_{2}\right)$, for example, is nearly axial, whereas for $\mathrm{Ni}(\mathrm{I})(\mathrm{STPP})\left(2,4-\mathrm{Me}_{2} \text { py }\right)_{2}$, with two axial 2,4-lutidine ligands, the $g$ values are very close to those of $\mathrm{MCR}_{\text {red2 }}$. A similar behavior is found for the $\mathrm{Ni}(\mathrm{III})(\mathrm{CTPP})$ complexes, where again, depending on the axial ligands, the $\mathrm{g}$ matrix reflects approximately tetragonal symmetry or can be highly rhombic. In all these compounds the rhombicity was attributed to a ground state described as a linear combination of $\mathrm{d}_{x 2-y 2}$ and $\mathrm{d}_{z 2}$ orbitals.

The $\mathrm{MCR}_{\text {red2 } 2}$ nitrogen with the smaller hyperfine interaction $\left(A_{\text {iso }}=13.8 \mathrm{MHz}\right)$ observed in the HYSCORE experiments is tentatively assigned to the pyrrole nitrogen of ring $\mathrm{A}$ of the cofactor $\mathrm{F} 430$ in $\mathrm{MCR}_{\mathrm{red} 2}$ (see Fig. 5). Ring $\mathrm{A}$ is more flexible than the rings $\mathrm{B}, \mathrm{C}$, and $\mathrm{D}$, which are $\pi$ conjugated. In $\mathrm{MCR}_{\mathrm{red} 2}$ we therefore assume that pyrrole ring $\mathrm{A}$ is slightly bent out of the plane of the macrocycle. This, together with the influence of the axial ligands, would lower the symmetry and cause a strong mixing of the d orbitals of the ground state. That this ${ }^{14} \mathrm{~N}$ hyperfine coupling represents an interaction with the nitrogen of the glutamine is unlikely, since the glutamine is assumed to coordinate via its oxygen to the $\mathrm{Ni}$ ion and the nitrogen is remote $(\sim 0.4 \mathrm{~nm}$ from the $\mathrm{Ni}$ ion in $\left.\mathrm{MCR}_{\text {silent }}\right)$. Consequently, the isotropic hyperfine coupling of the glutamine nitrogen is expected to be much 
Table $2{ }^{14} \mathrm{~N}$ hyperfine and nuclear quadrupole parameters of the pyrrole nitrogens of the "corphin" macrocycle of $\mathrm{MCR}_{\mathrm{red} 2}$ in comparison with other MCR forms and $\mathrm{Ni}(\mathrm{I})$ complexes

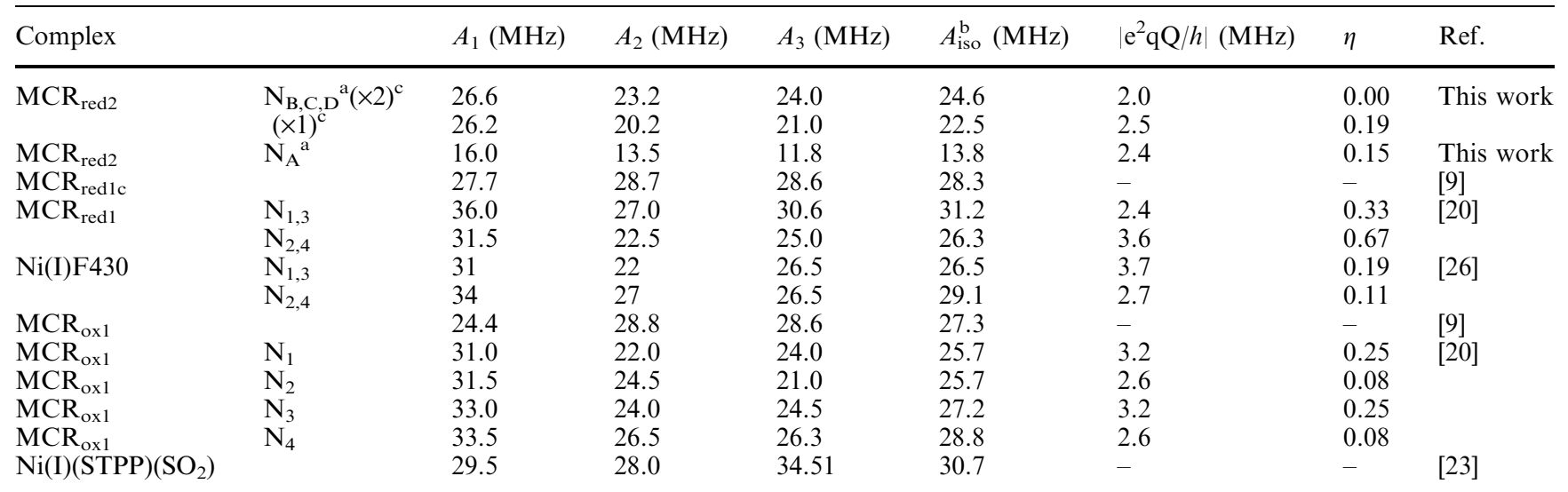

${ }^{\text {a }}$ Labels A, B,C, D indicate the pyrrole nitrogens of the macrocycle (see Fig. 5). Estimated errors are $\Delta A_{i}= \pm 0.7 \mathrm{MHz}, \Delta \mid \mathrm{e}^{2} \mathrm{qQ} /$ $h \mid= \pm 0.5 \mathrm{MHz}, \Delta \eta= \pm 0.2$

${ }^{\mathrm{b}}$ Calculated value, $A_{\text {iso }}=\left(A_{1}+A_{2}+A_{3}\right) / 3$

${ }^{\mathrm{c}}$ The two equivalent hyperfine couplings most likely come from the nitrogens in ring $\mathrm{B}$ and $\mathrm{D}$ since they are geometrically opposite (see Fig. 5), and the third hyperfine coupling would then be due to the nitrogen in ring $\mathbf{C}$. In the simulations, both the $\mathbf{A}$ and $\mathbf{Q}$ tensors are rotated with respect to the $\mathbf{g}$ matrix. For the two equivalent

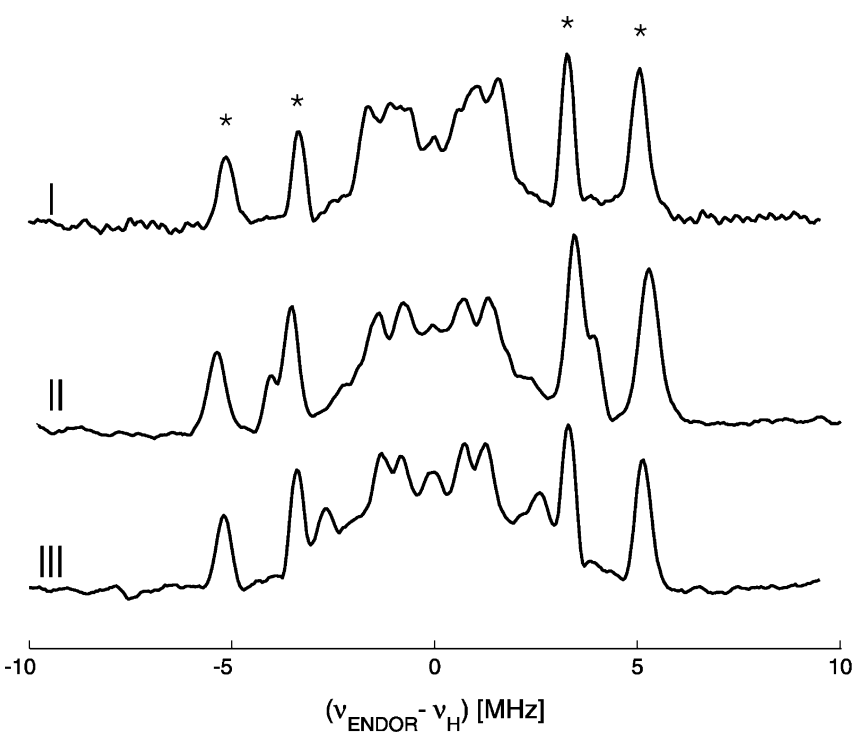

Fig. 4 Q-band proton Davies-ENDOR spectra at the observer positions indicated in Fig. 1. The asterisks label signals from protons with approximately isotropic hyperfine couplings. The spectra are centered around the ${ }^{1} \mathrm{H}$ Larmor frequency $\left(v_{\mathrm{H}} \approx\right.$ $48 \mathrm{MHz})$

smaller than $A_{\text {iso }}=13.8 \mathrm{MHz}$. It is worthwhile emphasizing that both ENDOR and HYSCORE spectra recorded at field positions where only $\mathrm{MCR}_{\text {red } 1}$ is present did not contain a weaker coupled nitrogen. These data show that there is a significant difference in the spin density distribution and macrocycle geometry between $\mathrm{MCR}_{\text {red1 } 1}$ and $\mathrm{MCR}_{\text {red2 }}$. nitrogens $\left(A_{\text {iso }}=24.6 \mathrm{MHz}\right)$ the Euler angles are $[\alpha, \beta, \gamma]=[45,0$, $0]^{\circ}$, and for the third $\left(A_{\text {iso }}=22.5 \mathrm{MHz}\right)$ and weakest coupled nitrogen $\left(A_{\text {iso }}=13.8 \mathrm{MHz}\right),[\alpha, \beta, \gamma]=[135,0,0]^{\circ}$. For all four nitrogens the axes of the largest absolute nuclear quadrupole value point along $\mathbf{A}_{1}$ and the smallest absolute value along $\mathbf{A}_{3}$. The axes of the largest hyperfine couplings $\left(A_{1}\right)$ are expected to point along the $\mathrm{Ni}-\mathrm{N}$ bond; in this case the $\mathbf{g}_{1}$ and $\mathbf{g}_{2}$ axes approximately bisect the $\mathrm{N}$ '-Ni-N bond angles [28]

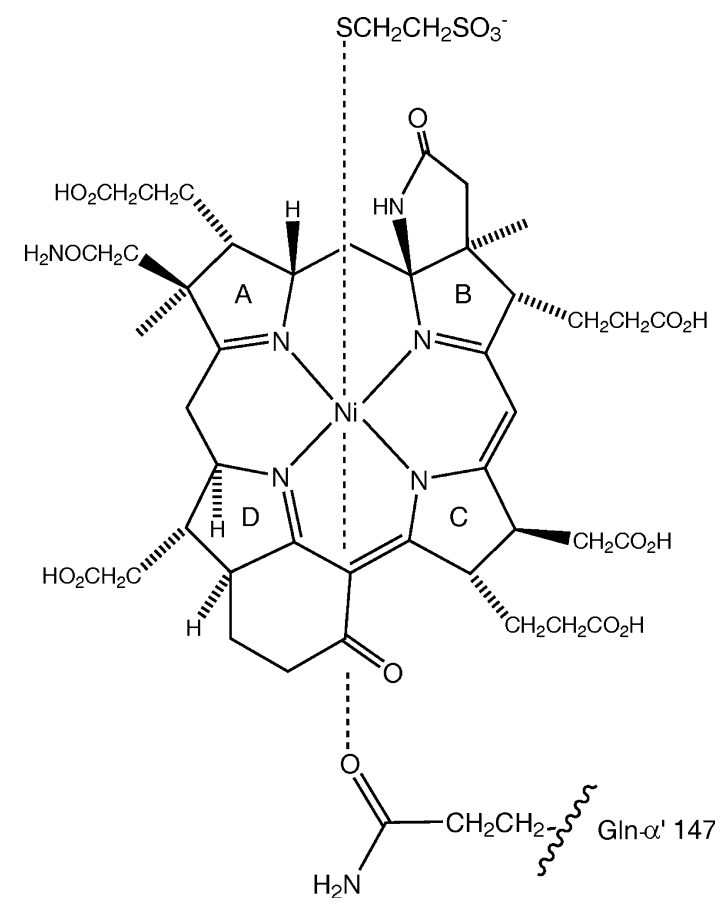

Fig. 5 Schematic drawing of the active site of ligation of methylcoenzyme $M$ reductase with $(\mathrm{H}) \mathrm{S}$-CoM coordinated via its sulfur, and glutamine $\alpha-147$ coordinated via its oxygen. The pyrrole rings are labeled $A, B, C$, and $D$

Based on the simulations of the $\mathrm{MCR}_{\text {red2 }}$ nitrogen ENDOR and HYSCORE spectra and on chemical arguments, we assume that the larger nitrogen hyperfine couplings arise from the three directly coordinated 
nitrogens of rings $\mathrm{B}, \mathrm{C}$, and $\mathrm{D}$ (see Table 2). The isotropic part of the hyperfine couplings $\left(A_{\text {iso }}=24.6\right.$ and $22.5 \mathrm{MHz})$ are smaller than the nitrogen hyperfine couplings found in $\mathrm{MCR}_{\text {red1 }}\left(A_{\text {iso }} \approx 28.5 \mathrm{MHz}\right)[9,14]$, $\mathrm{Ni}(\mathrm{I}) \mathrm{F} 430\left(A_{\text {iso }} \approx 27.8 \mathrm{MHz}\right)[26]$, and $\mathrm{Ni}(\mathrm{I})(\mathrm{STPP})\left(\mathrm{SO}_{2}\right)$ $\left(A_{\text {iso }}=30.7 \mathrm{MHz}\right)$ [23], reflecting the lower spin density on the macrocycle and the stronger $\mathrm{d}_{z 2}$ admixture.

From previous studies on related systems it is expected that the axis of the largest nitrogen hyperfine coupling will point along the Ni-N bond $[26,28]$, which for all four pyrrole nitrogens is in the plane spanned by $\mathbf{g}_{1}$ and $\mathbf{g}_{2}$ (see Table 2). This orientation implies that $\mathbf{A}_{3}$ and $\mathbf{g}_{3}$ are normal to the macrocycle plane, consistent with expectations from the $\mathrm{Ni}$ hyperfine tensor which has its largest value along the $\mathbf{g}_{3}$ axis $[9,10]$. The SOMO seems still to be predominantly $\mathrm{d}_{x 2-y 2}$ in character, since the largest nitrogen hyperfine couplings are closer to the values found for $\mathrm{MCR}_{\mathrm{red} 1}$ and $\mathrm{MCR}_{\mathrm{ox} 1}[20,27]$, as well as to $\mathrm{Cu}(\mathrm{II}) \mathrm{TPP}\left(\mathrm{d}_{x 2-y 2}\right.$ with $A \approx 40 \mathrm{MHz}$ [28]), than to complexes with a $\mathrm{d}_{z 2}$ ground state $\{$ e.g. CoTPP(py) with $A \approx 3 \mathrm{MHz}$ [29]\}. A more quantitative description of the ground state admixture and coordination geometry is not attempted here since it requires a more detailed description of the EPR parameters, including nickel and sulfur (from HS-CoM) data.

The isotropic proton hyperfine couplings observed in the ENDOR spectra of $\mathrm{MCR}_{\mathrm{red} 2}$ are tentatively assigned to the methylene protons of coenzyme $\mathrm{M}$, which is assumed to be axially coordinated to the nickel ion. Evidence in support of this assignment comes by comparing ${ }^{1} \mathrm{H}$ hyperfine couplings observed in $\mathrm{MCR}_{\text {red } 1}$ and the model compound $\mathrm{Ni}(\mathrm{I}) \mathrm{F} 430$. These complexes have a dominant $\mathrm{d}_{x 2-y_{2}}$ SOMO and no large and isotropic proton hyperfine couplings have been observed in the ENDOR spectra $(A \leq 4 \mathrm{MHz})$. This is consistent with the fact that the EPR spectrum of the essentially square planar complex $\mathrm{MCR}_{\text {red } 1}$ is not dependent on the presence of coenzyme $\mathrm{M}$, and that no evidence for axially coordinated ligands has ever been found for solutions of $\mathrm{F} 430$ in the $\mathrm{Ni}(\mathrm{I})$ oxidation state. The ground state of $\mathrm{MCR}_{\mathrm{red} 2}$ has a more pronounced $\mathrm{d}_{z 2}$ character, which decreases the spin density on the macrocycle (and the isotropic part of the macrocycle ${ }^{1} \mathrm{H}$ hyperfine couplings) relative to those of the complexes $\mathrm{MCR}_{\text {red1 }}$ and $\mathrm{Ni}(\mathrm{I}) \mathrm{F} 430$ with a dominant $\mathrm{d}_{x 2-y_{2}}$ ground state (see nitrogen couplings in Table 2). It is thus unlikely that the large and nearly isotropic ${ }^{1} \mathrm{H}$ couplings of $A_{\text {iso }}=6.7 \mathrm{MHz}$ and $A_{\text {iso }}=10.4 \mathrm{MHz}$ observed in $\mathrm{MCR}_{\text {red2 }}$ are due to protons of the macrocycle. Hence, the $\mathrm{C}_{\beta}$ protons adjacent to the spin-attracting sulfur of HS-CoM are candidates for the isotropic hyperfine couplings. These hyperfine couplings can probably be traced back to spin-polarization caused by spin density on the sulfur, with the couplings depending on the dihedral angles between the $\pi$ orbital of the $\mathrm{S}$ and the $\mathrm{CH}$ bond [30]. Similar isotropic hyperfine couplings have also been observed in type II blue copper proteins for cysteine $\beta$-protons [31] and in iron-sulfur clusters [32, 33].
To verify this hypothesis and to prove that HS-CoM is indeed an axial ligand of $\mathrm{MCR}_{\mathrm{red} 2}$, samples containing HS-CoM with a deuterated methylene group and/or ${ }^{33}$ S-labeled sulfur are in preparation ${ }^{1}$. These data, together with a more detailed analysis of the magnetic parameters of $\mathrm{MCR}_{\mathrm{red} 2}$, will be published elsewhere.

Acknowledgements This work was supported by the Swiss National Science Foundation, by the Max-Planck Society, and by the Fonds der Chemischen Industrie.

\section{References}

1. Thauer RK (1998) Microbiology 144:2377-2406

2. Pfalz A, Jaun B, Faessler A, Eschenmoser A, Jaenchen R, Gilles HH, Diekert G, Thauer RK (1982) Helv Chim Acta 65:828-865

3. Jaun B, Pfaltz A (1986) J Chem Soc Chem Commun 1327-1329

4. Ellefson WL, Whitman WB, Wolfe RS (1982) Proc Natl Acad Sci USA 79:3707-3710

5. Ermler U, Grabase W, Shima S, Goubeaud M, Thauer RK (1997) Science 278:1457-1462

6. Shima S, Goubeaud M, Vinzenz D, Thauer RK, Ermler U (1997) J Biochem (Tokyo) 121:829-830

7. Grabarse W, Mahlert F, Shima S, Thauer RK, Ermler U (2000) J Mol Biol 303:329-344

8. Grabarse W, Mahlert F, Shima S, Duin EC, Goubeaud M, Sima S, Thauer RK, Lamzin V, Ermler U (2001) J Mol Biol 309:315-330

9. Mahlert F, Grabarse W, Kahnt J, Thauer RK, Duin EC (2002) J Biol Inorg Chem 7:101-112

10. Mahlert F, Bauer C, Jaun B, Thauer RK, Duin EC (2002) J Biol Inorg Chem 7:500-513

11. Rospert S, Boecher R, Albracht SPJ, Thauer RK (1991) FEBS Lett 291:371-375

12. Goubeaud M, Schreiner G, Thauer RK (1997) Eur J Biochem 243:110-114

13. Rospert S, Voges M, Berkessel A, Albracht SPJ, Thauer RK (1992) Eur J Biochem 210:101-107

14. Schweiger A, Jeschke G (2001) Principles of pulse electron paramagnetic resonance. Oxford University Press, Oxford

15. Gromov I, Shane J, Forrer J, Rakhmatoullin R, Rozentzwaig Yu, Schweiger A (2001) J Magn Reson 149:196-203

16. Davies ER (1974) Phys Lett A 47:1-2

17. Höfer P, Grupp A, Nebenführ H, Mehring M (1986) Chem Phys Lett 132:279-282

18. Jeschke G, Rakhmatullin R, Schweiger A (1998) J Magn Reson 131:261-271

19. Madi ZL, Van Doorslaer S, Schweiger A (2002) J Magn Reson 154:181-191

20. Telser J, Horng YC, Becker DF, Hoffman BM, Ragsdale SW (2000) J Am Chem Soc 122:182-183

21. Salerno JC (1988) In: Lancaster JR Jr (ed) The bioinorganic chemistry of nickel. VCH, Deerfield Beach, Fla., USA, pp 53-102

22. Pinho D, Gomes P, Freire C, de Castro B (2001) Eur J Inorg Chem 6:1483-1493

23. Chmielewski P, Grzeszczuk M, Grazynski LL, Lisowski J (1989) Inorg Chem 28:3546-3552

24. Marganian CA, Vazir H, Baidya N, Olmstead MM, Mascharak PK (1995) J Am Chem Soc 117:1584-1594

25. Chmielewski PJ, Grazynski LL (1997) Inorg Chem 36:840-845

26. Telser J, Fann Y-C, Renner MW, Fajer J, Wang S, Zhang H, Scott RA, Hoffman BM (1997) J Am Chem Soc 119:733-743

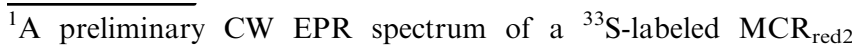
sample shows a line broadening along $\mathbf{g}_{3}$ that is due to hyperfine splittings from ${ }^{33} \mathrm{~S}$ 
27. Telser J, Davydov R, Horng YC, Ragsdale SW, Hoffman BM (2001) J Am Chem Soc 123:5853-5860

28. Brown TG, Hoffman BM (1980) Mol Phys 39:1073-1109

29. Van Doorslaer S, Bachmann R, Schweiger A (1999) J Phys Chem A 103:5446-5455

30. Atherton NM (1998) Electron paramagnetic resonance. The Royal Society of Chemistry, Cambridge
31. Epel B, Slutter CS, Neese F, Kroneck PMH, Zumft WG, Pecht I, Farver O, Lu Y, Goldfarb D (2002) J Am Chem Soc 124:8152-8162

32. Dikanov SA, Bowman M (1998) J Biol Inorg Chem 3:18-29

33. Mouesca JM, Rius G, Lamotte B (1993) J Am Chem Soc 115:4714-473 